\title{
Improving Data Dissemination in Multi-Hop Cognitive Radio Ad-Hoc Networks
}

\author{
Mubashir Husain Rehmani ${ }^{1}$, Aline Carneiro Viana ${ }^{2}$, Hicham Khalife ${ }^{3}$, and \\ Serge Fdida ${ }^{1}$ \\ 1 LIP6/UPMC Sorbonne Universités, France, \\ mubashir.rehmani@lip6.fr, serge.fdida@lip6.fr \\ WWW home page: http://www.lip6.fr/ \\ 2 INRIA, France, \\ aline.viana@inria.fr \\ WWW home page: http://www.inria.fr/ \\ 3 LaBRI/ENSEIRB, Université de Bordeaux, France, \\ hicham.khalife@labri.fr \\ WWW home page: http://www.labri.fr/
}

\begin{abstract}
In this paper, we present SURF, a distributed channel selection strategy for efficient data dissemination in multi-hop cognitive radio ad-hoc networks (CRNs). SURF classifies the available channels on the basis of primary radio unoccupancy and the number of cognitive radio neighbors using the channels. Through extensive NS-2 simulations, we compare the performance of SURF with three related approaches. Simulation results confirm that SURF is effective in selecting the best channels for efficient communication and for highest dissemination reachability in multi-hop CRNs.
\end{abstract}

Key words: multi-hop cognitive radio networks, channel selection, data dissemination

\section{Introduction}

Data dissemination is commonly defined as the spreading of information to multiple destinations through broadcasting. The main objective is to reach the maximum number of neighbors with every sent packet. In this communication scheme, no routing is required, thus neither routing tables nor end-to-end paths are maintained. Among different applications where data dissemination can be useful, we focus in this work on networking scenarios where providers disseminate nonurgent messages with limited cost and complexity through the network, such as: services, updates (e.g., new code to re-task a provided service), or any kind of publicity message. However, guaranteeing reliability of data dissemination in wireless networks is a challenging task. Indeed, the characteristics and problems intrinsic to the wireless links add several issues in the shape of message losses, collisions, and broadcast storm problem, just to name a few. Particularly in the context of Cognitive Radio Ad-Hoc Wireless Networks (CRN) 1], where 
channels for transmission are opportunistically selected, reliability is difficult to achieve. This is due to the inherent features of such networks. First, in addition to the already known issues of wireless environments, the diversity in the number of channels that each cognitive node can use adds another challenge by limiting node's accessibility to its neighbors. Second, Cognitive Radio (CR) nodes have to compete for the residual resources left by the Primary Radio (PR) nodes on many channels and use them opportunistically. Besides, CR nodes should communicate in a way that do not disturb the reception quality of PR nodes by limiting CR-to-PR interference.

Due to lack of centralized entity and the difficult coordination between CR nodes, the selection of a common channel by CR transmitters and receivers is a challenging task in multi-hop CRNs. In this context, we argue that the data dissemination reachability in such networks can be improved if (1) the chances for both transmitter and receivers, selecting the same channel for operating is increased and (2) the use of the best channel in terms of communication opportunities is prioritized. A lot of works have been carried out for dynamic channel management in CRNs. These approaches focus on single-hop CRNs 2 3. 4, 5, the presence of central entity, or the coordination with PR nodes in their channel selection decision. A proposal/solution related to our approach is Selective Broadcasting (SB) [6], however, SB requires more than one transceiver, resulting in bigger and more complex devices, as for military applications 7 . Besides, transmissions over a set of channels without considering the PR activity may increase the probability of interference with PR nodes.

In this paper, we propose SURF, a distributed and intelligent channel selection strategy for multi-hop CRNs. SURF classifies the available channels and uses them efficiently to create a multi-hop CRN, connected with high probability. The classification is done on the basis of PR unoccupancy and the number of CR neighbors using the channels. The objective of every CR node is to select the best channel ensuring a maximum connectivity and consequently, allowing the largest data dissemination reachability in the network. Hence, solutions should on the one hand select channels having low primary radio nodes (PRs) activities and achieve reliability by selecting spectrum bands that have high number of CR neighbors on the other hand. Moreover, SURF keeps track of previous wrong channel state estimation and accordingly adapts future channel selection decision. Usually channel selection strategies provide a way for nodes to select channels for transmission. SURF however, endues CR nodes to select best channels not only for transmission but also for overhearing. As a result, both sender and receiver tune with high probability to the same channel for effective and reliable data dissemination.

Through extensive NS-2 simulations, we show that SURF is effective in selecting the best channels for efficient communication. SURF offers the highest dissemination reachability in multi-hop CRN when compared with three other approaches i.e., Random, Highest Degree, and Selective Broadcasting []. Additionally, thanks to SURF, the amount of collision with the PR nodes is considerably reduced. In fact, $\mathrm{SURF}$ protects the $\mathrm{PR}$ nodes during channel selection 
decision. The simplicity and decentralized nature of SURF makes it usable in ad-hoc CRNs deployed to convey services, updates, or any kind of publicity messages.

The remainder of this paper is organized as follows: we discuss system model and assumptions in Section 2] We then give a general overview of SURF in Section 3 Section 4 and 5 deal with the description of SURF. Performance analysis is done in section $[$ section 7 discuss related work, and finally, section 8 concludes the paper.

\section{System Model and Assumptions}

We consider a Cognitive Radio Ad-Hoc Network, which is composed of a set of PR nodes and a set of CR nodes [8. In this type of network setting, we assume that no centralized network entity is available. Such entity could facilitate CR nodes in different network operations like spectrum sensing, channel selection decision etc. Instead, we consider these previous tasks are performed by the $\mathrm{CR}$ nodes themselves. We assume that $\mathrm{CR}$ nodes are equipped with a single transceiver, where a single channel can be selected at a time and used exclusively for transmission or overhearing. Such operating mode reduces the operational cost of the CR device 9 and avoids potential interference between co-located transceivers due to their close proximity [10]. We consider the set of $C$ total frequency channels. CR nodes can communicate using licensed or unlicensed bands, whichever bands are available. The use of licensed bands by cognitive radio nodes is however, only possible when the bands are idle, i.e. unoccupied by the PR nodes. We denote by idle the temporal availability of a channel. In some cases, it can happen that a CR node starts a transmission in the same time when PR becomes active. Since, we consider here CR transmissions should not generate harmful interference at PR receivers [11, CRs shall interrupt their transmissions.

We assume that spectrum sensing and the detection of the PR signal are not performed by SURF. These tasks are responsibility of the spectrum sensing block 12, which in turn provides PR spectrum unoccupancy information. In this case, SURF will work on the list of available channels resulted from the spectrum sensing. We further assume that SURF requires the information about CR neighbors and this neighbor discovery is not the part of SURF. CR neighbors can be discovered by using a Common Control Channel (CCC) 13 or with any other neighbor discovery mechanism, such as [14].

\section{Channel Selection Strategy SURF}

\subsection{Rationale}

SURF is a packet-based channel selection scheme for data dissemination and not a routing algorithm. SURF classifies available channels on the basis of primary 
radio unoccupancy and the number of $\mathrm{CR}$ neighbors using the channels. More precisely with SURF, every CR node autonomously classifies available channels based on the observed PR-unoccupancy over these channels. This classification is then refined by identifying the number of CRs over each channel. The best channel for transmission is the channel that has the lowest PR activity and a highest number of CR neighbors. Indeed, choosing a channel with few CRs increases the probability of having a disconnected network. Practically, every CR after classifying available channels, switches dynamically to the best one and broadcasts the stored message. Moreover, SURF tries to learn with previous wrong channel state estimation. This learning process allows better tuning the future estimations and helps CR nodes to recover from their bad channel selection decisions.

Additionally, CRs with no messages to transmit implement the SURF strategy in order to tune to the best channel for data reception. Clearly, using the same strategy implemented by the sender increases the chance that receivers in close geographic areas select the same used-to-send channel for overhearing. This is also due to the fact that, intuitively, it is likely that CRs in the sender's vicinity have the same PR unoccupancy. Hence, channels available to a CR sender are also available, with high probability, to its neighbors 15. In this way, SURF increases the probability of creating a connected topology. Once a packet is received, every $\mathrm{CR}$ receiver undergoes again the same procedure to choose the appropriate channel for conveying the message to its neighbors.

\subsection{Channel's Weight Calculation Formula}

SURF strategy classifies channels by assigning a weight $P_{w}^{(i)}$ to each observed channel $i$ in the channel set $C$. Thus, every cognitive radio running SURF, locally computes the $P_{w}^{(i)}$ using the following equation:

$$
\forall i \in C: P_{w}^{(i)}=P R_{u}^{(i)} \times C R_{o}^{(i)}
$$

$P_{w}^{(i)}$ describes the weight of a channel $i$ and is calculated based on the unoccupancy of PR (i.e. $P R_{u}^{(i)}$ ) and CR occupancy (i.e. $C R_{o}^{(i)}$ ) over channel $i$ (c.f. section 4 and section 5 . Then, the channels are ranked according to their weights and the best channel (i.e., the one providing highest $P_{w}^{(i)}$ ) is selected.

Practically, the computed weight in Eq. (11) increases with the PR unoccupancy and the CR occupancy. These two behaviors are directly related to the two objectives the SURF strategy needs to satisfy. The major objective of protecting the ongoing PR activity is mapped as a function of PR unoccupancy. The higher the probability of channel being in OFF state, i.e. $P R_{u}^{(i)}$, the higher the weight will be. The second objective of increasing connectivity is implemented in the second term of Eq. (11). More precisely, the weight increases with the number of $\mathrm{CR}$ neighbors i.e. $C R_{o}^{(i)}$. We now discuss each objective in detail. 


\section{Primary Radio Unoccupancy}

The primary radio activity, i.e. presence or absence of the PR signal, can be modeled as continuous-time, alternating ON/OFF Markov Renewal Process (MRP) [16], 17]. The authors in [18 validate this model for the presence of the PR signal. Fig. 1 illustrates the wireless channel model. The ON state, i.e. busy state, indicates that the channel is currently occupied by the PR node, while the OFF state, i.e. idle state, indicates that the channel is currently unoccupied by PRs.

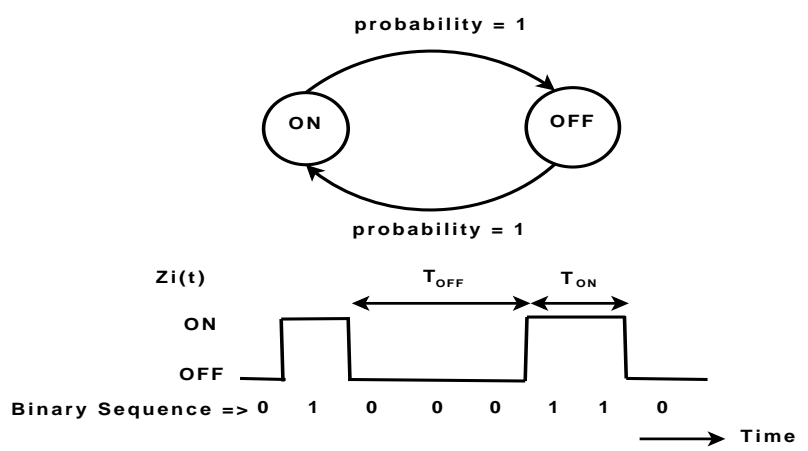

Fig. 1. Wireless channel model: Alternating Markov Renewal Process for PR activity.

As in [16, 19], we consider the channels ON and OFF periods are both exponentially distributed with p.d.f. $f_{X}(t)=\lambda_{X} \times e^{-\lambda_{X} t}$ and $f_{Y}(t)=\lambda_{Y} \times e^{-\lambda_{Y} t}$ respectively. Since our goal is to select the channel that will be unoccupied at time $t$, we only consider $P_{O F F}(t)$, the probability that the channel $i$ will be in OFF state at time $t$. Therefore $P_{O F F}(t)$, is calculated as:

$$
P_{O F F}(t)=\frac{\lambda_{X}}{\lambda_{X}+\lambda_{Y}}+\frac{\lambda_{Y}}{\lambda_{X}+\lambda_{Y}} e^{-\left(\lambda_{X}+\lambda_{Y}\right) t}
$$

The best channel at time $t$ is the one that has very high probability of being in OFF state. It may be possible that the next estimated channel state mismatches with the real state of the channel. This leads to bad channel selection decision and causes harmful interference to PR nodes. Next, we detail how the learning of previous wrong estimations are used to tune future estimations.

\subsection{Recovery from Bad Channel Selection Decisions}

The main challenges we deal with in this paper reside in making efficient and reliable channel selection decisions on-the-fly and in recovering from bad channel selection decisions. Clearly, keeping track of wrong channel state estimations can help CR nodes to recover from their bad channel selection decisions, which ultimately enhance the reliability and the performance. To achieve this goal, 
Table 1. Estimated and Current States of the Channel.

\begin{tabular}{|c|c|c|c|}
\hline Event & Estimated State & Current State & Probability \\
\hline \hline \multirow{2}{*}{$P_{U M}$} & OFF & ON & $P_{M D}$ \\
\cline { 2 - 4 } & ON & OFF & $P_{F A}$ \\
\hline
\end{tabular}

nodes maintain the history of their wrong channel state estimations and the observed current state of the channels. CR nodes then use this history to calculate the probability of unsuccessfully matched state $P_{U M} . P_{U M}$ is defined as the probability that the estimated channel state mis-matches with the actual channel state or simply how often the channel states estimation become erroneous. CR nodes then give the feedback of $P_{U M}$, while calculating the next channel state (cf. Fig. 2). More specifically, the accuracy of the SURF state estimation of channels depends upon the estimated state and the measured current state of the channel. Table. 1 provides the possible combinations between these two values.

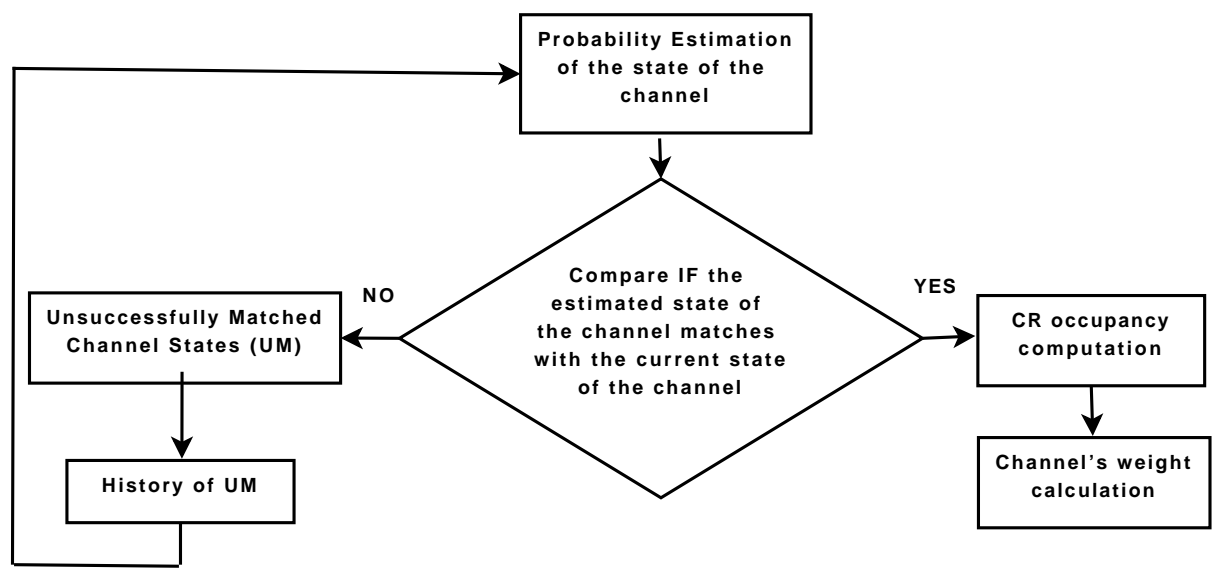

Fig. 2. Flow chart showing the corrective measure taken by CR nodes in the case of detection of unsuccessfully matched channel states i.e. $P_{U M}$.

The probability $P_{U M}$ is expressed as: $P_{U M}^{(i)}=\frac{x_{n t}}{N}$, where $x_{n t}$ is the number of times the estimated channel state does not match with the actual channel state i.e. how often the channel states estimation was erroneous, and $N$ is the number of coin flips. We further decompose $P_{U M}^{(i)}$ into $P_{M D}^{(i)}$ and $P_{F A}^{(i)}$ as: $P_{U M}^{(i)}=$ $\frac{x_{n t}}{N}=P_{M D}^{(i)}+P_{F A}^{(i)}$, where $P_{M D}$ refers to the Probability of Miss-Detection and occurs when $P_{O F F}$ estimation is $\mathrm{OFF}$ and the observed current channel state is ON. In $P_{M D}, \mathrm{CR}$ node declares the busy channel as unoccupied. This will lead to harmful interference with PR nodes. Whereas, $P_{F A}$ is the Probability of False-Alarm and occurs when $P_{O F F}$ estimation is $\mathrm{ON}$ and the observed current channel state is OFF. In $P_{F A}$, CR node declares that the unoccupied channel is 
Table 2. Wireless channel parameters used in the simulations.

\begin{tabular}{c|c|c|c|c|c|c|c|c|c|c}
\hline & Ch 1 & Ch 2 & Ch 3 & Ch 4 & Ch 5 & Ch 6 & Ch 7 & Ch 8 & Ch 9 & Ch 10 \\
\hline \hline$\lambda_{X}$ & 1.25 & 0.4 & 1 & 0.4 & 0.5 & 2 & 1 & 0.18 & 0.5 & 0.67 \\
\hline$\lambda_{Y}$ & 0.67 & 2 & 1 & 0.33 & 1 & 0.29 & 0.25 & 2 & 1.33 & 0.5 \\
\hline
\end{tabular}

busy. This will lead to refraining CR nodes from transmitting and thus, wasting precious spectrum opportunity.

Consequently, the lower the $P_{U M}(t)$, the more accurate will be the channel state estimation. Putting things together, we estimate the primary radio unoccupancy $P_{O F F}^{*}(t)$, which considers the probability of unsuccessfully matched state during the channel state estimation, as follows:

$$
P R_{u}^{(i)}=P_{O F F}^{*}(t)^{(i)}=P_{O F F}^{(i)}\left(1-P_{F A}^{(i)}\right)+P_{M D}^{(i)}\left(1-P_{O F F}^{(i)}\right)
$$

In the case of a perfect channel estimation (i.e., $P_{F A}=0$ and $P_{M D}=0$ ), $P_{O F F}^{*}(t)=P_{O F F}(t)$. In the presence of channel estimation errors, the probability of channel $(i)$ being in OFF state is given by Eq. (3). Note that when the channel has high weight but at time $t$ is occupied, SURF reacts (i) by not transmitting the packet on the best weighted channel, (ii) by selecting the next best weighted channel for packet transmission/overhearing, and (iii) by recomputing $P_{F A}$ and $P_{M D}$. Also note that when all the channels are occupied, no message is sent.

\section{Cognitive Radio Occupancy}

CR occupancy reflects the number of CR neighbors, which means the number of $\mathrm{CR}$ nodes using the channel $i$ at time $t$. In SURF, special consideration is given to selecting those channels that have higher number of CR neighbors. Higher number of $\mathrm{CR}$ neighbors provides good level of network connectivity and consequently increases the transmission coverage of $\mathrm{CR}$ nodes. The CR occupancy $C R_{o}^{(i)}$ of channel $i$ is estimated as: $C R_{o}^{(i)}=C R_{n}^{(i)}$, where, $C R_{n}^{(i)}$ is the number of $\mathrm{CR}$ neighbors using the channel $i$. As mentioned earlier, SURF requires information about $\mathrm{CR}$ neighbors, which can be discovered by using a Common Control Channel (CCC) [13] or with any other neighbor discovery mechanism, such as [14.

\section{Performance Analysis}

We analyze the performance of SURF through extensive simulations. We show here some results and we ask readers to refer to 21 for more detailed analysis. We enhanced the Cognitive Radio Cognitive Network (CRCN) patch 22] of NS2 to include the PR activity model. Practically, each channel alternates between ON and OFF states with rate parameters $\lambda_{X}$ and $\lambda_{Y}$ respectively (cf. Table 2).

We compare SURF with random strategy $(\mathrm{RD})$, highest degree strategy (HD) and selective broadcasting, proposed in [6] with multiple transmissions (SB). In 
$\mathrm{RD}$, channels are randomly selected to be used by $\mathrm{CR}$ nodes for transmission and/or overhearing, without any consideration to the ongoing PR and CR activity over these channels. HD approach only considers CR activities and is inspired by SB approach. In HD, CR nodes select the highest CR degree channel for transmission and overhearing, without any consideration of PR activity. The highest degree channel covers, consequently, the highest number of neighbors in the available list of channels. In SB, each CR node calculates a minimum set of channels, Essential Channel Set (ECS), for transmission that covers all its geographic neighbors, without considering the PR unoccupancy. In SB, a CR node transmits on multiple channels in round-robin fashion present in the ECS list, until all neighbors are covered. Note that in [6] nothing is mentioned about how nodes overhear over the channels. Therefore, we consider nodes select for overhearing the highest degree channel from their ECS list only. If more than one option is available, a random choice for transmission/overhearing is performed among those channels with the same degree.

Since, our goal is to efficiently disseminate the data and to protect the PR nodes from harmful interference, we define three performance metrics:

1. Harmful Interference Ratio (HIR): This metric is defined in order to capture the notion of collision with PR nodes. HIR is defined as the ratio of the total number of times the channel is found to be occupied by PRs after the channel selection decision over total number of times the channel selection occurs.

2. Average Delivery Ratio: This metric measures the data dissemination process. It represents the ratio of packets received by a particular CR node over total packets sent in the network.

3. Ratio of Accumulative CR Receivers: This metric also evaluates the data dissemination process. It is defined as the average ratio of accumulative CR receivers per hop over the accumulative effective neighbors per hop. Accumulative CR receivers per hop are the number of CR receivers per hop that successfully received the message, while accumulative effective neighbors per hop are the CR neighbors that selects the same channel for overhearing as the sender node used for transmission. By accumulative ratio we mean: at each new hop $n$, the receivers and effective neighbors of all previous hops $l<n$ are summed up to the ones at hop $n$.

The transmission range of $\mathrm{CR}$ nodes is set to $R=250 \mathrm{~m}$. The number of CR nodes is fixed to $\mathrm{N}=100$ and CRs are randomly deployed within a square area of $a^{2}=700 \times 700 m^{2}$. Simulations run for 1000 seconds. Total 1000 packets are sent, where each packet is sent by a randomly selected source node after every 1 second. All results are obtained with a confidence interval of $95 \%$.

We consider $5(\mathrm{Ch}=5)$ and $10(\mathrm{Ch}=10)$ total number of channels, that allow varying the neighborhood density $d_{\text {avg }}$ between 11.3 (when $\mathrm{Ch}=5$ ) and 20.1 (when $\mathrm{Ch}=10$ ). Note that this density is computed after the spectrum sensing provides the list of available channels and before the CRs select the channel to transmit/overhear. In this case, it is worth mentioning that, at the following simulation studies, the neighborhood density varies in function of the CRs' channel 


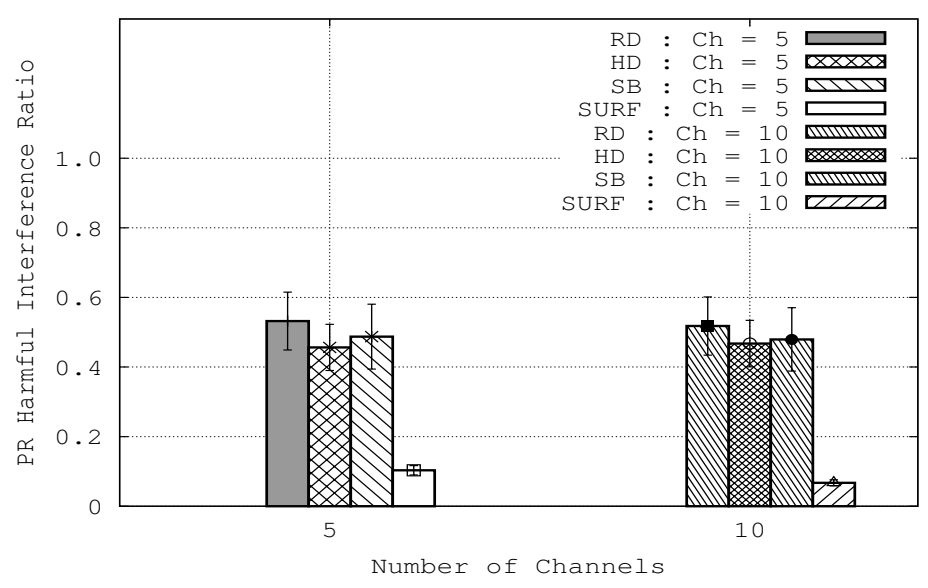

Fig. 3. PR harmful interference ratio for RD, HD, SB and SURF.

selection and is lower than the above ones. TTL is introduced to disseminate the message in the whole network. It is the maximum number of hops required for a packet to traverse the whole network, i.e., $\left\lceil\frac{2 a}{R}\right\rceil$, and is set to $T T L=6$ in our simulation scenario. Details on the used wireless channel parameters can be found in Table. 2] which were measured by authors in [19.

In summary, at each packet transmission event, the PR unoccupancy per channel $i,\left(P R_{u}^{(i)}\right)$, is calculated by each CR node. Then, each CR node locally computes the CR occupancy $\left(C R_{o}^{(i)}\right)$ and the weight $\left(P_{w}^{(i)}\right)$ of each channel $i$. The channel with the highest weight is then selected for transmission and/or overhearing. The message dissemination phase then starts, during which a randomly selected $\mathrm{CR}$ node disseminates the message on the selected channel by setting a TTL at the message. CR neighbor nodes that are on the same channel will overhear the message, decrease the $T T L$, redo the spectrum sensing, select the best available channel, and disseminate the message to the next-hop neighbors until $T T L=0$.

Harmful Interference Ratio Fig. 3 compares the harmful interference ratio for the four strategies i.e. $\mathrm{RD}, \mathrm{HD}, \mathrm{SB}$ and $\mathrm{SURF}$, for $\mathrm{Ch}=5$ and $\mathrm{Ch}=10$. It can be clearly seen in the figure that SURF, as expected, causes less harmful interference to $\mathrm{PR}$ nodes, compared to RD, HD, and SB. This is primarily because, when using SURF, CR nodes select those channels that have very high probability of being in OFF state, reducing thus PR interference. Note that in SURF, if all channels are occupied, the CR transmission will not take place. Thus, the lower HIR value for SURF in Fig. B is shown only to represent the cases where all channels were occupied by PRs and a probable interference is to be caused if a transmission takes place. In addition, when the number of channels is low, i.e. $\mathrm{Ch}=5$, the value of HIR is higher than $\mathrm{Ch}=10$. This is due to the fact 


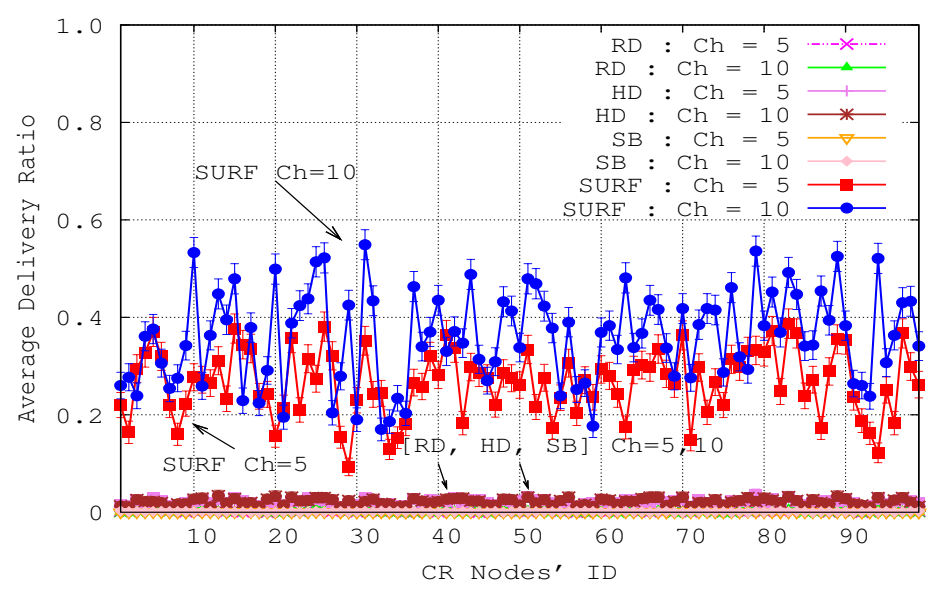

Fig. 4. CR Nodes' ID and average delivery ratio.

that a lower number of channels also reduces the chances for CR nodes finding PR-unoccupied channels for their transmission. As a result, SURF protects PR nodes, by reducing the amount of collisions with primary radios.

Average Delivery Ratio Fig. 4 compares the average delivery ratio of RD, HD, $\mathrm{SB}$ and $\mathrm{SURF}$, for $\mathrm{Ch}=5$ and $\mathrm{Ch}=10$. SURF increases considerably the delivery ratio compared to the other solutions. In particular, for $\mathrm{Ch}=5, \mathrm{SURF}$ guarantees a maximum delivery ratio of approximately $40 \%$ compared to almost $0 \%$ in the case of RD, HD, and SB. And when $\mathrm{Ch}=10, \mathrm{SURF}$ allows some nodes to reach a maximum delivery ratio of $50 \%$, while in $\mathrm{RD}$, it is almost $0 \%$ and $2 \%$ in $\mathrm{HD}$ and $\mathrm{SB}$. In fact, $\mathrm{RD}, \mathrm{HD}$, and $\mathrm{SB}$, do not guarantee that the selected channel is unoccupied for transmission thus causing a severe decrease in the delivery ratio. While in SURF, the average delivery ratio is higher because CR nodes selects the channel that has higher $P_{O F F}^{*}(t)$ and higher CR neighbors. It is worth mentioning that the diversity in terms of available channels and PR activities, and the consequent lower neighborhood density after CRs local channel selection result in the creation of different topologies (i.e., dynamic neighborhood) at each transmission/overhearing of CR nodes. These issues make hard the achievement of a higher delivery ratio than SURF.

In order to better observe the impact on delivery ratio of such dynamic neighborhood, Fig. 5 shows the average delivery ratio per node ID for $\mathrm{Ch}=5$ and $\mathrm{Ch}=10$ when $\mathrm{PR}$ activity equals to 0 . Similarly, Table 6 summarizes the overall average delivery ratio of Fig 4 and Fig. 5 . The results attest the obtained low delivery ratios are mainly due to the creation of different topologies resulted from the multi-channel availability and distributed channel selection by CRs. More specifically, even when no PR competition exists, the maximum average delivery ratio is lower than $35 \%$. Nevertheless, it is worth to note that SURF is the approach less impacted by the PR activities: By intelligently taking profit of 


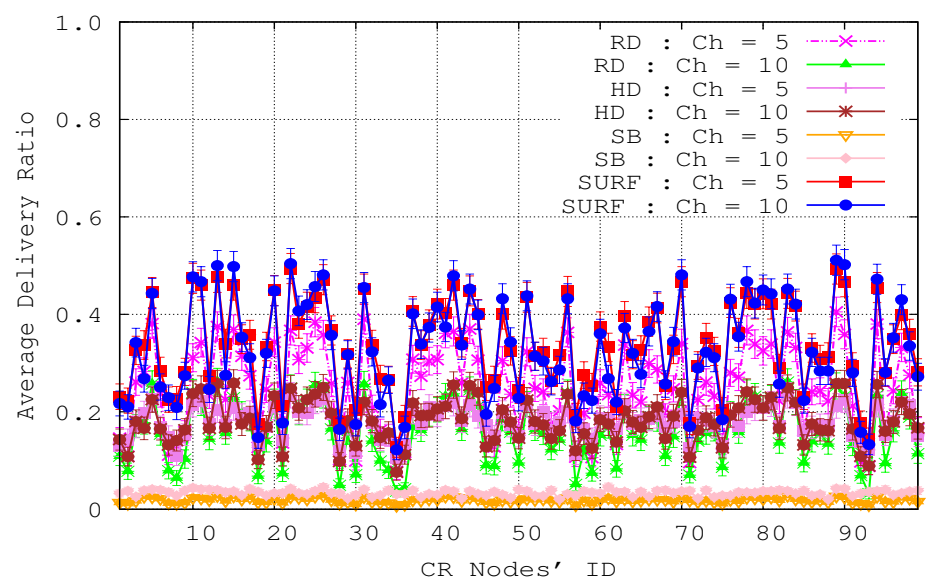

Fig. 5. CR Nodes' ID and average delivery ratio, when PR activity is zero.

Table 3. Overall average delivery ratio (in \%).

\begin{tabular}{|c|c|c|c|c|}
\hline & \multicolumn{2}{|c|}{$P R=0$} & $P R \neq 0$ & cf. Table 2] \\
\hline Strategy Name & $\mathrm{Ch}=5$ & $\overline{C h}=10$ & $\mathrm{Ch}=5$ & $\mathrm{Ch}=10$ \\
\hline $\mathrm{RD}$ & 0.25 & 0.16 & $\overline{0}$ & 0 \\
\hline HD & 0.18 & 0.18 & 0.02 & 0.02 \\
\hline SB & 0.02 & 0.03 & 0 & 0 \\
\hline SURF & 0.34 & 0.33 & 0.27 & 0.36 \\
\hline
\end{tabular}

channels availabilities, SURF is able to ensure a stable delivery ratio even when CRs transmission is competing with the PR ones.

Most importantly, it is worth noting that with the increase of the number of channels, SURF performance is also enhanced. This result is counterintuitive since adding more channels makes the synchronization between the sender and the receiver (i.e selecting the same channel) harder to achieve. However, by using the appropriate metric and mainly employing the same strategy at the sender and the receiver, SURF achieves better results when more channels are available.

Ratio of Accumulative Receivers Fig. 6] compares the ratio of accumulative receivers at each hop of communication (i.e until $T T L=0$ ) for RD, HD, SB, and SURF. SURF outperforms the 3 other techniques in all hops. At the $1^{\text {st }}$-Hop, due to the first transmission of the message, no collision is present. In this case, SURF provides a ratio of $95 \%$ receivers for $\mathrm{Ch}=10$ ( $80 \%$ for $\mathrm{Ch}=5)$, against $5 \%$ for RD, $12 \%$ for $\mathrm{HD}$, and $2 \%$ for SB. With the message propagation and its natural replication in the network, the probability of collisions increases and consequently, the receivers ratio at each new hop decreases, for all the strategies. Still, SURF provides a better dissemination ratio than other strategies. This is 


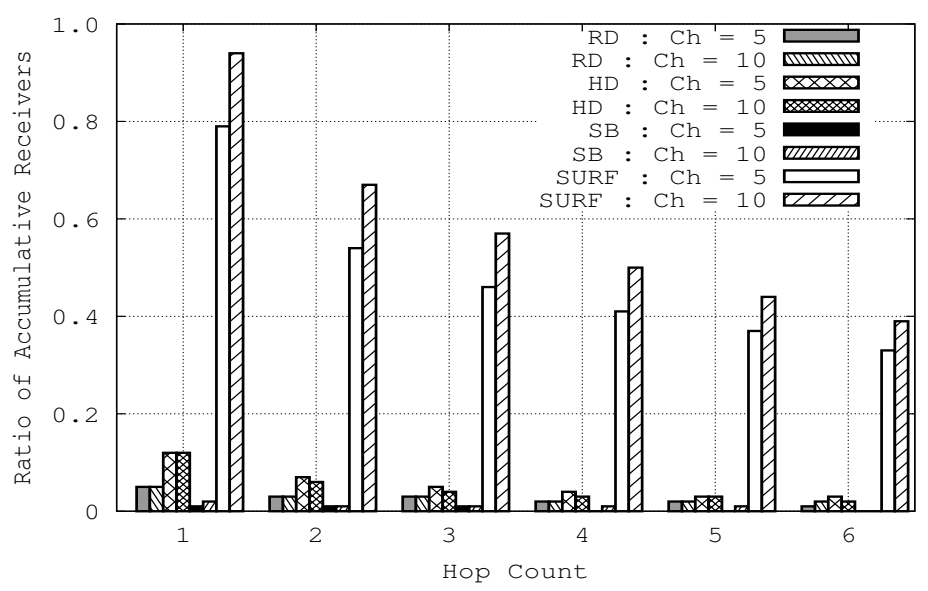

Fig. 6. Hop count and Ratio of accumulative receivers.

obtained thanks to the SURF channel selection, which selects channels providing high probability for good delivery as well as for good reception.

In summary, results in Fig. [ and Fig. [6 confirm that SURF can provide higher network reachability, suitable for increasing dissemination reliability in multi-hop cognitive radio networks.

\section{Related Work}

Recently, a lot of work has been carried out for dynamic channel management in cognitive radio networks [3, 4, 5, 15, 20, 25, 26. However, all these approaches focuses on single-hop cognitive radio networks and either requires the presence of any central entity or coordination with primary radio nodes in their channel selection decision. For instance, 3] proposed an efficient spectrum allocation architecture that adapts to dynamic traffic demands but they considered a singlehop scenario of Access Points (APs) in Wi-Fi networks. An approach that use non-continuous unoccupied band to create a high throughput link is discussed in [4. In [5], authors proposed a proactive channel selection strategy for TVbroadcast networks, which is single-hop and requires strong co-ordination with TV receivers in their channel selection decision.

A very few works has been done for channel selection in the context of multihop cognitive radio ad hoc networks so far [6, 23, 24. We compared SURF with Selective Broadcasting [6], in which each CR node calculates a minimum set of channels i.e. Essential Channel Set (ECS) for transmission that covers all its geographic neighbors, without considering the PR unoccupancy. In [23, 24], the authors proposed a dynamic resource management scheme for multi-hop cognitive radio networks. In fact, their approach is a route/channel selection 
for delay sensitive applications such a multimedia streaming, while SURF is a channel selection scheme for data dissemination and not for routing.

\section{Conclusion and Future Work}

We have introduced SURF, a channel selection strategy for reliable data dissemination in multi-hop CRNs. Simulation results in NS-2 confirmed that SURF, when compared to random-based, highest degree and selective broadcasting strategies, is effective in selecting the best channels. Furthermore, we show that unlike other solutions, SURF performance is enhanced with the increase of the number of existing channels. This is due to its intelligent selection mechanism. We intend in future to consider the traffic and data rates of $\mathrm{CR}$ nodes in the channel's weight calculation formula, as well as time needed to disseminate messages in the network.

\section{References}

1. I. F. Akyildiz, W.-Y. Lee, M. C. Vuran, and S. Mohanty, "Next generation/dynamic spectrum access/cognitive radio wireless networks: a survey," Computer Networks: The International Journal of Computer and Telecommunications Networking, vol. 50(13), pp. 2127-2159, 2006.

2. G. D. Nguyen, S. Kompella, and J. E. Wieselthier, A. Ephremides, Channel sharing in cognitive radio networks, in: MILCOM, 2010.

3. L. Yang, L. Cao, H. Zheng, and E. Belding, "Traffic-aware dynamic spectrum access," in Proceedings of The Fourth International Wireless Internet Conference (WICON 2008), Hawaii, USA, 17-19, Nov 2008.

4. H. Rahul, N. Kushman, D. Katabi, C. Sodini, F. Edalat, Learning to share: Narrowband-friendly wideband wireless networks, in: ACM SIGCOMM, 38(4), pp. 147-158, SEATTLE, WA, USA, 2008.

5. P. A. K. Acharya, S. Singh, H. Zheng, Reliable open spectrum communications through proactive spectrum access, in: TAPAS, 2006.

6. Y. R. Kondareddy, and P. Agrawal, "Selective Broadcasting in Multi-Hop Cognitive Radio Networks", IEEE Sarnoff Symposium, Apr 2008

7. O. Younis, L. Kant, K. Chang, K. Young, Cognitive manet design for mission-critical networks, IEEE Communications Magazine (2009) 64-71.

8. I. F. Akyildiz, W.-Y. Lee, and K. R. Chowdhury, "Crahns: Cognitive radio ad hoc networks," Ad Hoc Networks, 7(5), Jul 2009.

9. H. Harada, "A small-size software defined cognitive radio prototype," in Proceedings of the IEEE International Symposium on Personal, Indoor and Mobile Radio Communications (PIMRC), Cannes, France, 15-18 September 2008, pp. 1-5.

10. K. G. Shin, H. Kim, C. Cordeiro, and K. Challapali, "An experimental approach to spectrum sensing in cognitive radio networks with off-the-shelf ieee 802.11 devices," 4th IEEE Consumer Communications and Networking Conference, CCNC 200\%., 11-13 2007, pp. $1154-1158$.

11. H. Khalife, N. Malouch, and S. Fdida, "Multihop cognitive radio networks: to route or not to route," IEEE Networks, pp. 20-25, Aug 2009. 
12. T. Yucek and H. Arslan, "A survey of spectrum sensing algorithms for cognitive radio applications," IEEE Com. Surv. and Tutorials, 11(1), pp. 116-130, Mar 2009.

13. S. L. Loukas Lazos and M. Krunz, "Spectrum opportunity-based control channel assignment in cognitive radio networks," in 6th IEEE SECON, Rome, Italy, 22-26 June 2009.

14. C. Arachchige, S. Venkatesan, N. Mittal, An asynchronous neighbor discovery algorithm for cognitive radio networks, in: New Frontiers in Dynamic Spectrum Access Networks, 2008. DySPAN 2008. 3rd IEEE Symposium on, 2008, pp. $1-5$. doi:10.1109/DYSPAN.2008.78

15. Q. Zhao, L. Tong, A. Swami, and Y. Chen, "Decentralized cognitive mac for opportunistic spectrum access in ad hoc networks: A POMDP framewrok," IEEE JSAC, 25(3), pp. 589-600, Apr 2007.

16. A. W. Min and K. G. Shin, "Exploiting multi-channel diversity in spectrum-agile networks," in IEEE INFOCOM, Apr 2008.

17. W.-Y. Lee and I. Akyildiz, "Optimal spectrum sensing framework for cognitive radio networks," IEEE Trans. on Wir. Commun., 7(10), pp. 3845 -3857, Oct 2008.

18. S. Geirhofer, L. Tong, and B. M. Sadler, "Dynamic spectrum access in wlan channels: Emperical model and its stochastic analysis," in ACM TAPAS, Aug 2006.

19. H. Kim and K. Shin, "Efficient discovery of spectrum opportunities with mac-layer sensing in cognitive radio networks," IEEE Trans. on Mobile Comp., 7(5), pp. 533 -545 , May 2008.

20. D. Niyato, E. Hossain, Competitive spectrum sharing in cognitive radio networks: A dynamic game approach, IEEE Transactions on wireless communications 7(7), pp. 2651-2660, July 2008.

21. M. H. Rehmani, A. C. Viana, H. Khalife, and S. Fdida, "SURF: A distributed channel selection strategy for data dissemination in multi-hop cognitive radio networks," INRIA, Technical Report, 2011. [Online]. Available: http://hal.inria.fr/inria-00596224/en/

22. [Online]. Available: http://stuweb.ee.mtu.edu/ ljialian/

23. J. Zhao, H. Zheng, G. H. Yang, Distributed coordination in dynamic spectrum allocation networks, IEEE Trans. Veh. Tech., 58(2), pp. 941-953, Feb 2009.

24. H. P. Shiang, M. V. D. Schaar, Delay-sensitive resource management in multi-hop cognitive radio networks, IEEE DySpan, 2008.

25. C. Cordeiro, K. Challapali, D. Birru, and S. N. Shanka, "IEEE 802.22: An introduction to the first wireless standard based on cognitive radios," Journal of Communications, 1(1), pp. 38-47, Apr 2006.

26. M. Hoyhtya, S. Pollin, and A. Mammela, "Classificaiton-based predictive channel selection for cognitive radios," in IEEE ICC, May 2010. 